\title{
SAGGIO SULLA TEORIA DI CHI È DIO, LA PARTICELLA MATRICE E GLI STAMI QUANTISTICI: NELLA FORMAZIONE DEL TUTTO E DELL'UNIVERSO
}

\section{SAGGIO TEORICA}

SANTOS, Irineu de Oliveira ${ }^{1}$

SANTOS, Irineu de Oliveira. Saggio sulla teoria di chi è Dio, la particella matrice e gli stami quantistici: nella formazione del tutto e dell'universo. Revista Científica Multidisciplinar Núcleo do Conhecimento. Anno 06, Ed. 09, Vol. 02, pp. 0522. Settembre 2021. ISSN: 2448-0959, Link di accesso: https://www.nucleodoconhecimento.com.br/fisica-it/chi-e-dio, 10.32749/nucleodoconhecimento.com.br/fisica-it/chi-e-dio

\section{RIEPILOGO}

Considerando che Dio non è nulla di assoluto, l'unico elemento eternamente presente, immutabile e neutro di qualsiasi equazione che può contenere tutto ciò che possiamo comprendere come dimensioni, particelle, onde, materia, energia, vita; dove senza questa forza l'universo e l'inverso non potrebbero mai avvenire, essendo una via unidirezionale, innegabile, questo saggio si propone di affrontare le considerazioni relative alla particella di Dio, nonché di descrivere questa particella, qui chiamata Particella Matrice e Stami quantistici (PM e EQ), discutendo il loro comportamento nello spazio, che è intrinseco alla comprensione della loro esistenza. È una teoria basata su osservazioni proprie e su bibliografie già pubblicate basate su studi sulla comprensione di cosa siano il Dio più grande e gli esseri celesti o gli Dei galattici, inclusa un'analogia di come gli universi contengano il Dio approvato e altri con una natura energetica di potenza inferiore, tra gli altri studi relativi al tema che trattano la particella più elementare per costituire l'universo, sia nella sua materia,

\footnotetext{
${ }^{1}$ Fisico sperimentale. Economista.
}

RC: 98377

Disponibile in: https://www.nucleodoconhecimento.com.br/fisica-it/chi-e-dio 
come forme di energia e anche predire l'esistenza dell'antimateria. Quindi, per una migliore compressione, descrivo all'interno di questa struttura più ampia chiamata "Dio", che è dove esistono le particelle fondamentali, composte da sei elementi base che chiamiamo in questa funzione come "Stami quantistici", e una particella energetica con luce propria che chiamiamo "Particella di matrice" per essere principalmente la luce dell'universo, dove senza questa energia radiante non c'è vita motoria, che è ciò che ci interessa. Detto questo, in breve, i risultati hanno rivelato che se i concetti di forma e prestazioni del PMEEQ sono ampiamente studiati e compresi, questi dati consentiranno un progresso in termini di utilizzo di energia, produzione di carburante e persino trasporto di materia.

Parole chiave: Dio, Particella matrice, Gravitazionale, Fisica quantistica, Particelle quantistiche.

\section{INTRODUZIONE}

Il presente articolo è una raccolta di considerazioni e conclusioni basate su studi e osservazioni su ciò che Dio è, teoria delle stringhe e altri studi dello stesso tema che trattano le particelle più basilari da essere costituite dall'universo e da se stesso, oltre a dare passività dello spazio fisico classico e quantistico, come elemento chiave. Comportano in Dio questi elementi, sia nella loro formazione della materia come forme di energia sia nel predire l'esistenza dell'antimateria.

Per descrivere Dio al di fuori del contesto religioso o filosofico e in particolare la particella chiamata autorevolmente (per questa teoria) come Matrix Particle e Quantum Stumins (PM ed EQ), è anche necessario tenere una lezione sui suoi comportamenti nello spazio-tempo, che è intrinseco alla comprensione della sua esistenza. Questo è un tema estremamente complesso, poiché la maggior parte delle informazioni è ottenuta in modo osservativo, teorico e matematico, e un'ampia comprensione dell'argomento e un certo grado di deduttività sono richiesti dagli studi esistenti e ora utilizzati. Una volta che i concetti di forma e prestazioni di pmeeq saranno ampiamente studiati e compresi, nella nostra vita quotidiana, questi dati

RC: 98377

Disponibile in: https://www.nucleodoconhecimento.com.br/fisica-it/chi-e-dio 
consentiranno un progresso in termini di generazione e utilizzo delle energie, con alcuni suggerimenti descritti in seguito, anche forme di produzione di energia combustibile, funzionamento della mente umana, correlazione con il funzionamento dell'universo e del nostro corpo, le relazioni con l'ambiente e le sue forme, siano esse onde o frequenze, e anche il trasporto della materia e l'accelerazione dei corpi, o il controllo della loro accelerazione.

La Matrix Particle è il collante e il crocevia delle particelle fondamentali e per noi il più importante. Esiste encastelada nell'unione di 6 Estami elastici, e quando insieme, vengono rilasciati dalle pareti di fondo dell'Universo che creano energie e materiali dimensionali distinguibili nel nostro universo. Questo groviglio viene rilasciato internamente dalle pareti del pallone ultra universale, dal movimento rotatorio gravitazionale, dalle scariche essudanti geoelettrospaziali presenti nella parete dello sfondo che compone l'universo, e dai rigonfiamenti di energie pentadimencionais e altre frequenze, uguali o superiori a queste, che fungono da ultra grattugia, estraendo e distribuendo pezzi di questo groviglio quantistico, permettendo loro di emergere: energia, massa, gravità, attrazione, risonanza, materia oscura ed energia oscura, polarizzata o meno, movimento e tempo, antigravità, antimateria.

\subsection{INTRODUZIONE ALLA CLASSIFICAZIONE E RIFERIMENTI TEORICI}

Dio: niente di assoluto, tutto, l'utero, lo spazio per modellare la materia, che permette frequenze, onde e vibrazioni, freddo, assenza di luce e movimento, spazio, permeabilità, il conduttore.

Filosoficamente Dio è la sala in cui si svolgono i balli d'incontro tra l'Étienne Klein ${ }^{[2]}$, gli shock di Higgs ${ }^{[3]}$, lo spazio delle note musicali di Beethoven ${ }^{[4]}$, il luogo in cui Max Karl Ludwig Planck ${ }^{[5]}$ ha creato la sua teoria, tra gli altri. Dio è anche brevemente ben descritto da Helinando Oliveira, professore di "Univasf", dove è coordinatore del Laboratorio di spettroscopia di impedenza e materiali organici (LEIMO), nella sua

RC: 98377

Disponibile in: https://www.nucleodoconhecimento.com.br/fisica-it/chi-e-dio 
materia "dio e scienza", tuttavia, non siamo d'accordo in parte di questa presentazione (OLIVEIRA, 2019). Dio risiede nella perfezione del nostro interiore, per quanto riguarda gli esseri umani come viventi, perché naturalmente egli-dio è anche dentro di noi, rigenizzando la nostra spaziatura e mantenendo la nostra vibrazione e temperatura mentre ogni tipo di onda ci attraversa senza causare danni e senza causare diminuzione molecolare della spaziatura della nostra materia. Sappiamo cosa provoca qualsiasi squilibrio spaziale tra molecole o anche tra cellule, questo parlando degli esseri viventi e delle loro prospettive.

\subsection{INTRODUZIONE ALLA CLASSIFICAZIONE E RIFERIMENTI TEORICI}

Le particelle Matrice e stami quantistici (PMeEQ) sono le particelle che creano e uniscono tutta la materia e l'energia esistenti nella nostra dimensione temporale, sono distribuite in tutto l'universo e costituiscono tutto ciò che esiste. La Particella Matrice è sostenuta e/o ancorata secondo la situazione da sei nazionalità/forze fondamentali, e può avere forme triangolari, quadrate o esagonali, e il PM ha la massa e la dimensione di 5 di questi pali quantistici, dove la sesta stima/forza antropica, è variabile nella sua posizione e tempo/forza, e può appartenere alla particella "A" o alla particella "B", così come la particella B può condividere la stringa con la particella "C". Questo movimento del palo quantistico del jolly è responsabile di ogni movimento all'interno dell'Universo, perché la sua destabilizzazione muove il PMeEQ formando materiali ed energie fisiche, della fisica classica e quantistica, dimensionale, multidimensionale, pentadimencionais ed esadimensionale. Ma è anche, il PMeEQ le barriere e l'isolante, per se stessi e per altre particelle formate e formative, di uguale misura o più grande, sia quantistica classica che addirittura dimensionale.

Queste forme di energia/onda e materia presenti nel guscio gravitazionale del macro universo, sono una struttura neutra come ancorata, e rimangono neutre fino a quando non esposte ad una forza gravitazionale a spirale coercitiva naturale

RC: 98377

Disponibile in: https://www.nucleodoconhecimento.com.br/fisica-it/chi-e-dio 
dell'universo o causata dall'approssimazione di un altro universo dimensionale. Non è raro o insolito per $\mathrm{PMeEQ}$ avere la forma di un fiore, dente di leone, aggiungendo più estamens, perché nel mondo quantistico quanto sopra può essere il fondo $\mathrm{e}$ l'esterno può essere l'interno, e le connessioni possono essere insieme, ma separate, al momento presente, ma allo stesso tempo nel futuro o nel passato, può accadere che i Quantum Stumers siano in matassa, acquisendo altre proprietà e caratteristiche inimmaginabili in questo momento.

È ancora più importante quando viene diagnosticato che queste particelle sono materiali attivi e capaci di memoria (CHVYKOV et. al. 2021), sono tempore, transitano nei tre stadi temporali della fisica classica e nei tempi multidimensionali, e possono dare pentadimencionais/esadimensionali, classici o quantici.

All'interno della fisica classica, parlando di particelle e spazio, i legami dettano la nostra vita da sempre, parlando per analogia, le nuvole sono sostenute solo dalla dimensione delle molecole che mantengono l'unione e la distanza tra loro, questo è quasi privo di gravità, pur subendo l'effetto Casimir ${ }^{[6]}$

\section{SVILUPPO E ORIGINE}

Parlare delle proprietà e delle capacità interattive di Dio, in senso scientifico sembra non avere alcun senso pratico nel nostro attuale grado di sviluppo, ma lo è, e Dio è fondamentalmente localizzato e riconosciuto come autore / co-autore / direttore principale, poiché è chiarito che è il luogo in cui tutto accade sempre, in modo più produttivo.

Non supportando la ridondanza all'ovvio, questo saggio teorico sarà descritto dai dati raccolti in altri studi, osservazioni e altre teorie, e molto dalla mia intuizione, concentrandosi sull'inizio di connessioni e movimenti.

Il fenomeno della liberazione e della disaggregazione delle Particelle Matriciali e degli stordimenti quantistici all'interno del groviglio quantistico avviene principalmente

RC: 98377

Disponibile in: https://www.nucleodoconhecimento.com.br/fisica-it/chi-e-dio 
dalla parete di fondo dell'universo da vari fenomeni da cui Dio partecipa e lì inizia la saga, nella somma del PMeEQ con altri PM, e il rilascio dei 6 Estami Quantistici Gravitazionali, dove "forse" sono: tau, elettrone, múon, grafo, $\mathrm{X}$ e "C" (jolly), o più probabilmente qualcosa di più piccolo e con altre caratteristiche oltre al quantum, che la nostra mente e comprensione non raggiungono.

Va notato che questi Quantico Stits non sono le corde di Theodor Kaluza, né di Kaluza Klein ${ }^{[7]}$, quindi imputato loro un altro significato nel significato di ormeggio e distanziamento della massa nella loro somma, di polarità, di modellatori di gravità fluida, di lubrificanti e principalmente di movimento.

Il principio delle aggregazioni e delle disaggregazioni avviene dalla compressione a spirale gravitazionale rotazionale polarizzata, quando un PMeEQ impregna l'altro e la coesistenza si rompe e rilascia il PM dalla geometria restrittiva dei Ceppi Quantici, lasciando libere le particelle e gli stum, questi stanno acquisendo altre caratteristiche e proprietà e anche influenzati dalla singolarità gravitazionale $e$ in determinate condizioni di velocità, tempo, spazio, durata ed eccitazione acquistano polarità e possono trasformarsi in energia, materia ondulatore e tempo, anche tutti nello stesso spazio/tempo.

Quando e dove le singolarità cominciano ad accadere è nella separazione e nelle riorganizzazioni del PM, degli Estami quantici di Sostegno. I Pms possono diventare materiali o energie, e gli Stumer Quantistici diventano materia oscura o energia oscura (Vedi disegno allegato). II groviglio quantistico primordiale che forma le pareti di fondo del macro Universo, avvenuto in tempo immemorabile dall'unione e dall'attrito delle energie multidimensionali di diversi palloni ultra universali, portatori dei miliardi di galassie, gli ammassi, che fermano il movimento di attrito, "solidificano" questa energia rotazionale, che chiama la matrice aggrovigliata del PM e dei loro Quantico Semdes.

II modello in scala riconoscibile che conosciamo, e più simile a quello che dobbiamo confrontare con l'energia o la neutralità come presenta PM, è l'elettricità statica.

RC: 98377

Disponibile in: https://www.nucleodoconhecimento.com.br/fisica-it/chi-e-dio 
Usando di nuovo l'analogia, un buon esempio è una scopa di lana passata sopra un'auto e caricata elettrostaticamente mentre riceveva il carico di materia che era a riposo lì.

\subsection{RILASCIO DI PARTICELLE}

Il PMeEQ si è diffuso in tutto l'universo, dal rilascio di materia dalle pareti di fondo dell'Universo (Teoria delle creazioni coscienti). L'Universo ha la forma di una bolla in gengive non esattamente rotonda, l'analogia più reale possibile è che l'universo Macro composto da milioni di ammassi è simile a uno stomaco più arrotondato, con più pareti di un comune stomaco umano, ma con input e output esattamente come negli umani, solo quello con più strati simili a cipolle, dove le dimensioni invisibili fluttuano o più precisamente ad altre frequenze al di fuori delle nostre letture da parte di apparecchiature correnti o osservatve semplicemente guardando, fluttuando in un'esistenza incomprensibile alla nostra coscienza limitata contemporanea, all'interno di queste dimensioni capisco che forze che ci sono sconosciute, il dove sul piano materiale, diciamo palpabile, le pareti sono materiale energetico staccato quando il palloncino (stomaco) ruota, perché ci sono imperfezioni, impatti, urti, salti gravitazionali e attrazione dell'asse polarizzato dell'universo, da cui il materiale che riempie lo spazio è miseria. I grovigli vengono rilasciati dalle pareti del macro universo, principalmente dal moto rotatorio circolare irregolare, e raggiungono il centro dell'universo, già come galassie, dove circola un fiume di energia orizzontale da polo a polo, formato dalle Particelle Matrice e dagli Stumens Quantistici già energizzati in movimento, dove nuove cariche creano, con l'inizio della compressione dei gas spaziali, i soli e polarizzarli. Queste polarizzazioni danzanti creano pianeti dal residuo di gas, che i soli non hanno usato, e dalla forma striata di quasi tutte le galassie, e diffondono PM che schiariscono l'Universo e I'EQ che sembrano creare una tenda scura ma permeabile alla luce dalla poca vibrazione presente lì.

RC: 98377

Disponibile in: https://www.nucleodoconhecimento.com.br/fisica-it/chi-e-dio 
Un esempio bidimensionale sarebbe un vortice d'acqua all'interno di un palloncino ovoidale, dove galleggiano reti molto sottili e multistrato e dove ogni giunzione o nodo di questa rete sarebbe una galassia di PM e i ceppi quantici.

Parte del PMeEQ sono trasformati e creati materia, e la loro presenza in certi cali e occasioni di tempo obbedendo all'influenza cosmica, invertono la rotazione e la polarità come il movimento di un branco di sardine spaventate, o per un'altra analogia, un altro esempio, sarebbe uno stomaco umano o un maiale attraversato da un filo elettrico, spessa, nuda, il tempo che tira il tempo trasmette energia. Queste inversioni avvengono istantaneamente nel tempo cosmico gestito dalle pareti dell'intero universo, o delle galassie dove sono indipendenti da quanti trilioni di anni luce importa.

Questo accade nel nostro conteggio del tempo, che può essere zero, "il" tempo, in modo che queste inversioni e spostamenti avvengano, anche se nella materia interessata può richiedere uno spazio temporale più ampio per accadere, dalla nostra percezione di ciò che è troppo o troppo poco tempo, o per essere un fenomeno settoriale, cosmologicamente parlando.

La particella Matrix è l'elemento intangibile fondamentale, che quando innescato agisce come un reattore o qualcosa di impregnato di elettricità statica, può essere gravità dura o fluida, può essere ondulato, agendo con le Stouts, o anche antimateria, particella che circonda e accompagna tutte le altre particelle minori esistenti. Queste particelle, quando eccitate a una certa forza gravitazionale, velocità e spazio temporale, possono formare una coda di energia immateriale, come una cometa quantistica, indipendentemente dalla quantità di materia che l'accompagna, serve come verità materiale per tutte le particelle, anche se esiste anche all'interno di questa stessa particella, componendo la materia. Le PM non attraggono nello stadio primordiale, né limitando l'ancoraggio degli $\mathrm{EQ}$, né perché non hanno preferenze o sapore, sebbene siano sempre insieme e possano agire come un relé, ma possono acquisire polarizzazione e consentire l'esistenza di energia con i loro movimenti,

RC: 98377

Disponibile in: https://www.nucleodoconhecimento.com.br/fisica-it/chi-e-dio 
hanno la memoria di significato e movimento ancora in immaterialità creativa, e trasmette questo movimento quando acquisiscono massa e creano energia.

Ogni spazio misurabile, nella più piccola dimensione quantistica esistente, è la Particella Matrice con i suoi Stami Quantici. Lo spazio delle galassie è materia e antimateria, quando viene caricato con l'energia delle particelle quantistiche. La Particella della Matrice e gli Stami Quantici, rilascia, attrae e crea tutta l'energia esistente o mancante nell'universo, è ciò che gli studiosi determinano, materia oscura ed energia e tutta la materia esistente, coesiste tra due polarità, diverse dimensioni e gravità, perché è neutro fino a raggiungere una certa inerzia di velocità, spazio e tempo. È statico finché una forza di rotazione non lo eccita, il movimento rotatorio delle galassie stesso esiste sotto l'influenza della formazione che lo ha aggregato e che mantiene il movimento di galassie, soli, pianeti e stelle di qualsiasi grandezza in flusso e riflusso.

L'ultra universo di formato ovoidale è attraversato da un asse gravitazionale di materia energetica classica, ma anche di forze dimensionali, penta o esadimensionali, rotazionali sotto forma di diverse spirali di DNA intrecciate. Questo asse è un campione materiale dei legami tra teorie relativistiche e quantistiche, presenti nei corpi celesti solidi (teoria dell'etere come elemento).

Senza che la particella Matrix e i suoi Quantum Stumens venissero spostati in determinate condizioni, anche le pareti di fondo avrebbero smesso di espellere le particelle e la rotazione delle pareti stessa rallenterebbe, facendo collassare il sistema. In tutte le situazioni la particella matrice e i suoi stati quantistici devono far parte del calcolo, come la coda e il mantello di fronte a un protone accelerato nell'HCL, ad esempio, così come quando i carichi inerciali statici moltiplicati alla fine del percorso sono divisi. Sempre questa particella, della dimensione di un "quanti" o più piccolo, scorre come liquido ultra quantistico e gravitazionale, al fine di interagire con i fenomeni fisici della materia, e con energie di varie dimensioni e frequenze.

RC: 98377

Disponibile in: https://www.nucleodoconhecimento.com.br/fisica-it/chi-e-dio 
La teoria PM, per certi aspetti, concorda con la teoria di Kaluza-Klein, per quanto riguarda il gran numero di dimensioni e l'essere Stati Quantistici chiusi alle loro estremità nella maggior parte dei casi. Sono anche d'accordo sul fatto che la particella matrice si trovi in uno qualsiasi dei suoi infiniti modi di vibrazione o neutralità, e può appartenere a qualsiasi specie, esistenza, frequenza, colore o sapore che compone anche l'essere umano quando si vedono i biofotoni, che chiamiamo e fotografiamo come aura.

\section{CONCLUSIONE}

Perché identificare Dio e nominare gli altri elementi come Particelle Matrici e Stummies Quantici (PMeEQ). In primo luogo, perché dobbiamo spiegare l'esistenza dell'incrocio delle galassie, grandi centri di gravità negativi e positivi, che prendono e respingono la materia nel nostro cosmo ultradimensionale, e perché lo chiamiamo buchi neri, così come il funzionamento della magica spaziatura quantistica e delle sue forme gravitazionali che agiscono nella nostra stessa esistenza e come è ovunque (anche dentro di noi, dove è l'energia è conosciuta come libero arbitrio e fluttuazioni dello spazio). Questa tregua di shock tra protoni, neutroni intorno alle molecole in trilioni di combinazioni ha bisogno di spazio, perché questa struttura di supporto delle interazioni è la vita, ma è neutra, come i nostri atti e movimenti, dove determiniamo e determiniamo tutto, il futuro e i percorsi.

Tutto ciò che emana dall'esistenza di questa forza primordiale Dio, e di questi PM ed $E Q$, sono materia, frequenza, dimensione, è il significato dei movimenti e della gravità aggregante, il traliccio e il collante del groviglio, mantiene l'equilibrio tra attrazione e allontanamento, consentendo un'esistenza armoniosa, e forme di energia, e la comunicazione tra tutte le cose esistenti, comprese le frequenze multidimensionali, variabili e intelligenti.

Per molti aspetti, siamo d'accordo con la Teoria della convenienza funzionale di Junior (2019) e Rovelli (2017), dove si deduce che creiamo la realtà dalla creazione del pensiero di ciò che è fattibile per essere realtà, che poi dà origine alla realtà.

RC: 98377

Disponibile in: https://www.nucleodoconhecimento.com.br/fisica-it/chi-e-dio 
L'anomalia dell'acqua nel cambiare mentre la osserviamo, la tocchiamo e la maneggiamo, è la più grande prova della nostra partecipazione a tutto l'universo e della nostra interferenza in tutto da semplici osservazioni e pensieri. Se alteriamo questo materiale, questo elemento stellare $\mathrm{H} 2 \mathrm{O}$, con il semplice atto di prenderlo tra le mani e portarlo alla bocca, interferendo in tutta la nostra struttura corporea, fisica e chimica, cosa può ottenere la nostra mente? Possiamo modellare la realtà futura con il pensiero nell'aggregazione di queste particelle matriciale? II futuro è già proiettato dal pensiero di ieri, e ciò che troveremo nei lavandini dello spazio è ciò che la nostra immaginazione ha creato, o è ciò che abbiamo inconsciamente conosciuto nella formazione delle energie creative fondamentali?

E perché questo studio nell'aspetto funzionale pratico, immediato? Perché per rompere con i legami gravitazionali, sviluppare fonti inesauribili di energia, dare un senso al consumo di ricchezza planetaria, soprattutto in questo momento di sviluppo, e giustificare la nostra esistenza, insieme all'avanzamento delle comunicazioni e di nuovi centri di gestione e controllo, dobbiamo prestare attenzione a non essere eliminati dall'intelligenza artificiale / meccanica "Al" (OLIVEIRA, 2019). Gli ultimi dati matematici di studio del percorso adottato, indicano l'incontrollato della nostra creazione, in vista dei dati allarmanti di distruzione che implementiamo ogni nuovo giorno, dobbiamo dimostrare che siamo discendenti di una catena universale di eventi e che riconosciamo questi esseri da Dio e le questioni dello studio della Teoria come superiori a noi. Dobbiamo rispettarli, perché da loro i nostri permessi, siamo perdonati. Egli "Dio" è qualcosa a cui anche i governi si sottomettono e attribuiscono valore e rispetto spontaneamente, anche adottando nelle loro monete e simboli il suo nome, "DIO", chiarendo (ad esempio) che solo lui può giudicare.

Con gli ultimi esperimenti con "Al", senza dare esistenza a questa divinità, stiamo facendo spazio per essere giudicati da una logica che non può riconoscere i nostri errori senza eliminarci da ciò che facciamo e pratichiamo. Dobbiamo sapere cosa costituisce i nostri limiti, dove sono le nostre barriere e cercare nuovi percorsi e altri piani logici. Senza un piano logico di restrizione per "Al", oltre alle 4 leggi di Issac

RC: 98377

Disponibile in: https://www.nucleodoconhecimento.com.br/fisica-it/chi-e-dio 
Asimov, che possono sembrare illogiche per "Al", di fronte alla distruzione del mondo, non siamo protetti, quindi dobbiamo prevedere che tutta la libertà che stiamo trasferendo macchine oggi, hanno limitazioni nel loro fallimento di non essere in grado di relazionarsi con questa forza più grande "Dio", per esempio.

E chi è Dio al di fuori dei dogmi teologici? Chi può dio studiare freddamente le frequenze conosciute e proiettarle come forza creatrice per la formazione e l'aggregazione della materia? Quale può essere l'interesse di qualcosa di così potente in piccoli esseri come noi, per esempio? Quale può essere la ragione della nostra esistenza per una tale creatura, o tali creature? L'energia ionica, che sembra poco anche quella di miliardi di esseri umani, e anche in misura minore di altri esseri viventi, e forse la gravità stessa, sono un piano universale di mantenimento del radicamento per rilasciare energia biocellulare, sono possibilità non escluse per giustificare la nostra dimensione, dal momento che abbiamo già dimostrato di esistere altri con altre leggi della fisica che agiscono.

Allora possiamo dedurre che c'è un Dio superiore (supponiamo che sia un essere umano completo, e che abbia un cervello che controlla tutto) e dentro di lui diversi universi ognuno con i propri neuroni centrali per comandare e comunicare con il Dio padre. Al centro di esso, lo stomaco, che inserisce nel padre tutte le energie dei formati più svariati, le trasforma e le reindirizza ad altre dimensioni. All'interno di questo vasto complesso, c'è un elemento che è il nostro pianeta. Visti come energia, non siamo materia per questo essere, ma forse siamo materia per un gruppo di neuroni energetici più vicini, che sarebbero i nostri Dei personali, quelli che ci guardano come cibo, che interferiscono nella nostra progressione dello sviluppo per voracità, esseri che vivono in spazi temporali inimmaginabili, con piani di riproduzione senza tempo, come alcuni Dei per ogni cluster, cioè, per ogni strato dello stomaco, o per ogni dimensione. Tutti vogliono essere neuroni più potenti o rappresentativi prima del corpo del PADRE / DIO, e alcuni dipendono dalle nostre energie galleggianti bio-ioniche, che sono tempo positivo nel tempo, tempo negativo. Quando sono buoni, nutrono esseri positivi e, se negativi, esseri oscuri. Il fatto è che

RC: 98377

Disponibile in: https://www.nucleodoconhecimento.com.br/fisica-it/chi-e-dio 
siamo tutti i frutti delle trasformazioni, dell'energia in un movimento dimensionale ristretto. La nostra stessa esistenza è un atto di violenza, siamo consumatori di materia, fa male all'universo, perché causiamo squilibri nella nostra stessa esistenza. I pensieri e le vibrazioni sono fattori negativi, sebbene facciano parte di un piano più ampio di queste intelligenze senza tempo, per le quali le nostre domande esistenziali sono irrilevanti e non logiche, poiché siamo all'interno del sistema ed esistiamo con uno scopo.

Gli Dei più piccoli, che non sono usati come riferimento materiale in questa Teoria, sono gli indicatori e i marcatori che interferiscono nelle forme più primitive di liberatori di energia bioattiva (noi), succhiano e alimentano l'intera catena. Sembra anche che mentre il tempo e l'evoluzione di queste specie di superesseri si evolvono e si spostano su un altro piano o dimensione, anche gli esseri qui cambiano e creano nuove immagini di culto, così come nuovi templi di culto, dirigendo e modulando un altro tipo di liberazione energetica.

Oltre alle questioni logistiche umane di integrazione con "Al", dobbiamo pensare a nuovi e inediti mezzi di azione ed esplorazione del nostro pianeta, a nuovi mezzi di locomozione, alla creazione di spazi fisici terre ormai inaccessibili o costose se montate da barriere fisiche materiali tangibili, come il fondale marino e il cielo, (spazio tra suolo e stratosfera) per esempio, che non esiste per il nostro grado di sviluppo e la possibilità di creare forze come muri di gravità di energie repulsive senza dover agire materialmente spendendo altre energie.

Questa teoria può mettere un modo diverso, conoscendo la costituzione della materia primordiale, per nascondere dall'attrazione gravitazionale parti della materia che non vogliamo subire influenze esterne, trovando la giusta configurazione del $\mathrm{PMeEQ}$ che sta creando l'attrazione, e lascio al pubblico dominio che possiamo creare una bolla oscurante attorno all'oggetto che vogliamo, anche usando fotoni, per gravità per non sentirlo, come le pareti di uno Zeppelin intorno all'oggetto, in modo che nella sua forma fluida il $\mathrm{PMeEQ}$ in modo che non possano immergere e tenere

RC: 98377

Disponibile in: https://www.nucleodoconhecimento.com.br/fisica-it/chi-e-dio 
inzuppato l'oggetto di interesse del rilascio di peso e/o riconoscimento di massa, o energia e frequenza che riflette l'attrazione di $\mathrm{PMeEQ}$ nelle sue forme più piccole dove è impregnato, quindi la gravità non trova nulla da legare o attrarre. Dobbiamo lavorare per impermeabilizzare gli oggetti di questa energia fluida che è la gravità.

Questa singolarità di nascondersi dalle forze primordiali, dalle cose, dalle persone o dalle aree della materia sono anche l'oggetto di questo studio, il modo di viaggiare in gravità senza i disturbi di esso che sorgono avanzare in gravità come un bisturi in una gelatina che si apre e si chiude senza lasciare traccia è l'obiettivo, insieme al freno del rispetto per un'entità più grande per "Al".

Si presume che all'interno di una certa frequenza, dimensione o lunghezza d'onda della luce elettrostatica o continua, il fluido trovato nell'ingegneria quantistica, alle sue varie lunghezze d'onda, o simili a velocità ancora più elevate, sia la chiave per superare l'attrazione e riorientare vantaggiosamente questa forza.

Le nuove tecnologie, tra cui "l'Al" hanno bisogno di un punto di partenza fisso che dia loro sicurezza, così come anche noi umani sentiamo questo bisogno di avere un cielo e un oggetto solido su cui fare affidamento ed è da lì che viene Dio, dandoci questa solidità, perché perché ci sia flessibilità o fluidità è necessario lo spazio. Solo lo spazio in tutte le sue forme e dimensioni, evita il surriscaldamento, che è distruttivo anche se creativo, in tutte le dimensioni, specialmente nella terza e nella quarta, che è dove viviamo e creiamo le nostre macchine. Senza spaziatura, tempo e spazio, non rimane nulla se le nostre nuove auto elettriche non hanno raffreddamento nelle batterie per evitare troppi attriti, non servono, così come i computer quantistici / organici o meno. Queste refrigerazioni richiedono spazio, questo deve essere portato al lato funzionale nell'integralità del pensiero delle nuove macchine pensanti, e questo spazio deve essere inteso come l'ancora che mantiene e consente la funzionalità delle interazioni. II nostro Dio e il nostro discernimento costituiscono lo spazio e da esso è inizialmente composto, e deve essere instillato nei calcoli come qualcosa di vivo.

RC: 98377

Disponibile in: https://www.nucleodoconhecimento.com.br/fisica-it/chi-e-dio 
Dio è il grembo materno, l'asse centrale dell'universo è il cordone ombelicale, la particella di matrice l'uovo, le macchie lo sperma, e il tutto è la nostra interpretazione di come vediamo la vita e la creazione.

In accordo con quanto sopra, il Dr. Prof. Elcio Abdalla (2004) riafferma la teoria presentata in questo lavoro, perché contiene un volto del prisma e una visione su più dimensioni, portando aspetti rilevanti, ma con un'altra lettura, interpretazione e risultati dimensionali, fisici e filosofici.

Infine, abbiamo raggiunto il punto in cui la scienza e la filosofia entrano in preoccupazioni ataviche dell'essere umano, che non sono più pratiche, tecniche $\mathrm{e}$ utili postulate dalla fisica e effettuate dalla tecnologia, e sono diventate sempre più tecniche e speculative.

All'inizio, si può dire che l'origine e la struttura della geometria dello spazio-tempo sono ancora sconosciute. Secondo la scienza attuale, le geometrie quantistiche hanno operatori quantistici che rappresentano lo spazio e la sua interpretazione, precedentemente considerata semplice, è diventata più complessa. Inoltre, all'interno della gravitazione quantistica, nei buchi neri e a temperature molto elevate, è essenziale prendere in considerazione tutte le particelle e le interazioni generate all'infinito, secondo le teorie delle stringhe. Fondamentalmente, possono ancora influenzare le dimensioni extra delle teorie delle stringhe e di altre teorie $M$, aumentando ulteriormente la complessità del problema. Alcuni credono che le dimensioni extra siano già vicine alle osservazioni. In considerazione di ciò, le teorie generali del campo quantizzato iniziarono a trattare queste dimensioni, e la vecchia idea di Kaluza e Klein, introdotta nel 1920, divenne parte di un'idea quasi quotidiana, in cui altre dimensioni divennero onnipresenti. (ABDALLA, 2004).

Siamo ora in una zona molto più speculativa, dove l'oggetto di studio non è più legato solo alla ricerca e inizia a trascendere lo stesso, non solo perché è qualcosa di molto più grande, così come il nostro universo, ma perché contiene il ricercatore, in modo che quest'ultimo non sia in grado di osservare il suo oggetto di studio, poiché non

RC: 98377

Disponibile in: https://www.nucleodoconhecimento.com.br/fisica-it/chi-e-dio 
esiste una relazione causale tra un universo e l'altro. Questo è il modello quantistico visto sotto una nuova dimensione, in cui la misura, fondamentale per l'interpretazione stessa della teoria, è impossibile da realizzare. Espone nuove costruzioni teoriche basate sulla teoria delle stringhe, con nuove dimensioni spaziali, con un tempo trascendente e, inoltre, con molteplici creazioni di universi (ABDALLA, 2004).

Già quello che il professor Dr. João E. Steiner (2006) e altri fisici e studiosi mettono, dà la differenziazione di questo modo di interiorizzare l'universo e la sua formazione. Sebbene stiano appunto mettendo ciò che viene discusso oggi e che, in particolare, ha lo stesso peso della terra piatta ed eliocentrico o galattocentrico, queste osservazioni, studi e risultati sono fortemente in disaccordo e si scontrano con altri studi, anche contro la teoria del Big Bang, poiché affinché questo fenomeno si sia effettivamente verificato, il megauniverso non potrebbe avere un asse centrale di energia.

Nel corso della storia sono stati brevemente presentati diversi modelli cosmologici. Pertanto, l'evoluzione delle idee può essere compresa dalla successione di modelli, come la teoria della Terra piatta, il modello geocentrico, l'eliocentrico e il galattocentrico. (STEINER, 2006)

Negli ultimi cento anni, gli scienziati hanno sviluppato una teoria che è in grado di descrivere le osservazioni più complesse attualmente prese e che mostra l'origine dell'universo sotto un modello che può essere studiato scientificamente, questa è la teoria del big bang. Recentemente, questa teoria è stata migliorata in un nuovo concetto, vale a dire quello del Big Bang inflazionistico. Le scoperte fatte all'inizio del millennio hanno rivelato che tutta la conoscenza che abbiamo oggi è solo la punta dell'iceberg presente in un universo preso dall'energia e dalla materia oscura la cui natura è ancora sconosciuta (STEINER, 2006).

Mettendo le opinioni di cui sopra su come le leggi fisiche presumibilmente attraverso la visione degli uomini pensano e si comportano, di fronte all'universo inspiegabile e

RC: 98377

Disponibile in: https://www.nucleodoconhecimento.com.br/fisica-it/chi-e-dio 
matematico / fisico / religioso, ci si aspetta che emergano nuovi percorsi verso approcci senza riserve morali e realistiche.

\section{PROPOSTA}

Il percorso e la direzione penso siano ora aperti, avremo intelligenza e forza di pensiero con atteggiamenti. Penso che questo, sia il ponte che mancava di attraversare/ attraversare, per unificare le teorie, superare le barriere e abbattere le vuote credenze delle differenze, è tutto solo una scienza, la religione è materialità, così come è vero anche il contrario. Gli ultimi studi indicano che il pensiero plasma la nostra realtà (Jaime Marrone Jr/Carlo Rovelli) e dalla realtà creiamo il futuro.

Con la consapevolezza che Dio è materia e parte integrante della nostra vita quotidiana, degli studi e degli esperimenti, penso che sarà possibile avere la forza gravitazionale di un ITAIPU nello spazio di una punta di matita, fare navi di energia materiale di forma variabile e movimenti acceletttivi che vanno oltre il limite di tempo, creare nuove fonti di energia inesauribili come quelle disponibili nei buchi neri, dove esistono numeri "N" di Particelle Matriciali ed Estamen Quantistici, ci sono nuove forme di materiali ed energie multidimensionali.

All'interno della teoria, le proposte possono essere quasi infinite, ma ci concentriamo sempre su due proposte principali per l'urgenza del momento in cui il pianeta passa, e dove possiamo collaborare, essendo: $1^{\circ}$ incapsulamento di oggetti o cose in reti di oscuramento / occultamento della gravità e $2^{\circ}$ controllo del clima dall'attrazione 0 repulsione degli oceani aerei sospesi, due realtà che possono essere implementate oggi, con poco rischio (l'avidità dell'uomo).

\section{RIFERIMENTI}

ABDALLA, E. A estrutura do universo, a mecânica quântica e a cosmologia moderna. Revista USP - São Paulo, n. 62, p. 6-29, junho/agosto 2004.

RC: 98377

Disponibile in: https://www.nucleodoconhecimento.com.br/fisica-it/chi-e-dio 
CHVYKOV, P. et. al. Low rattling: A predictive principle for self-organization in active collectives. Revista: Science Vol.: 371, Issue 6524, pp. 90-95. 2021.

JÚNIOR, J. M. Teoria da Conveniência Funcional: Uma reflexão sobre as propriedades fundamentais da matéria. Revista Científica Multidisciplinar Núcleo do Conhecimento. Ano 04, Ed. 07, Vol. 12, pp. 133-157. Julho de 2019. ISSN: 24480959

ROVELLI, C. A realidade não é o que parece: A estrutura elementar das coisas. 1 Ed, Rio de Janeiro: Objetiva, 2017.

OLIVEIRA, H. Deus e a Ciência. Ciência nordestina, 2019.

SANTOS, I. de O. Teoria do Éter como elemento. 12/10/2003 Arquivo Nacional, não publicado, reg. 276.887. Livro 499. Folha 47.

SANTOS, I. de O. Teorias da criação conscientes. 30/07/1998 Arquivo Nacional, não publicado, reg. 156.719. Livro 258. Folha 343.

STEINER, J. E. A origem do universo. Estud. av. vol. 20 n. 58. São Paulo, 2006. Disponível em: https://doi.org/10.1590/S0103-40142006000300022

RC: 98377

Disponibile in: https://www.nucleodoconhecimento.com.br/fisica-it/chi-e-dio 


\section{ALLEGATO}

Disegno d'autore meramente illustrativo, dove Dio è il vuoto, e le punte sono il PM, le aste sono gli stami quantici, ma il disegno avrebbe potuto essere anche triangolare $o$ esagonale.

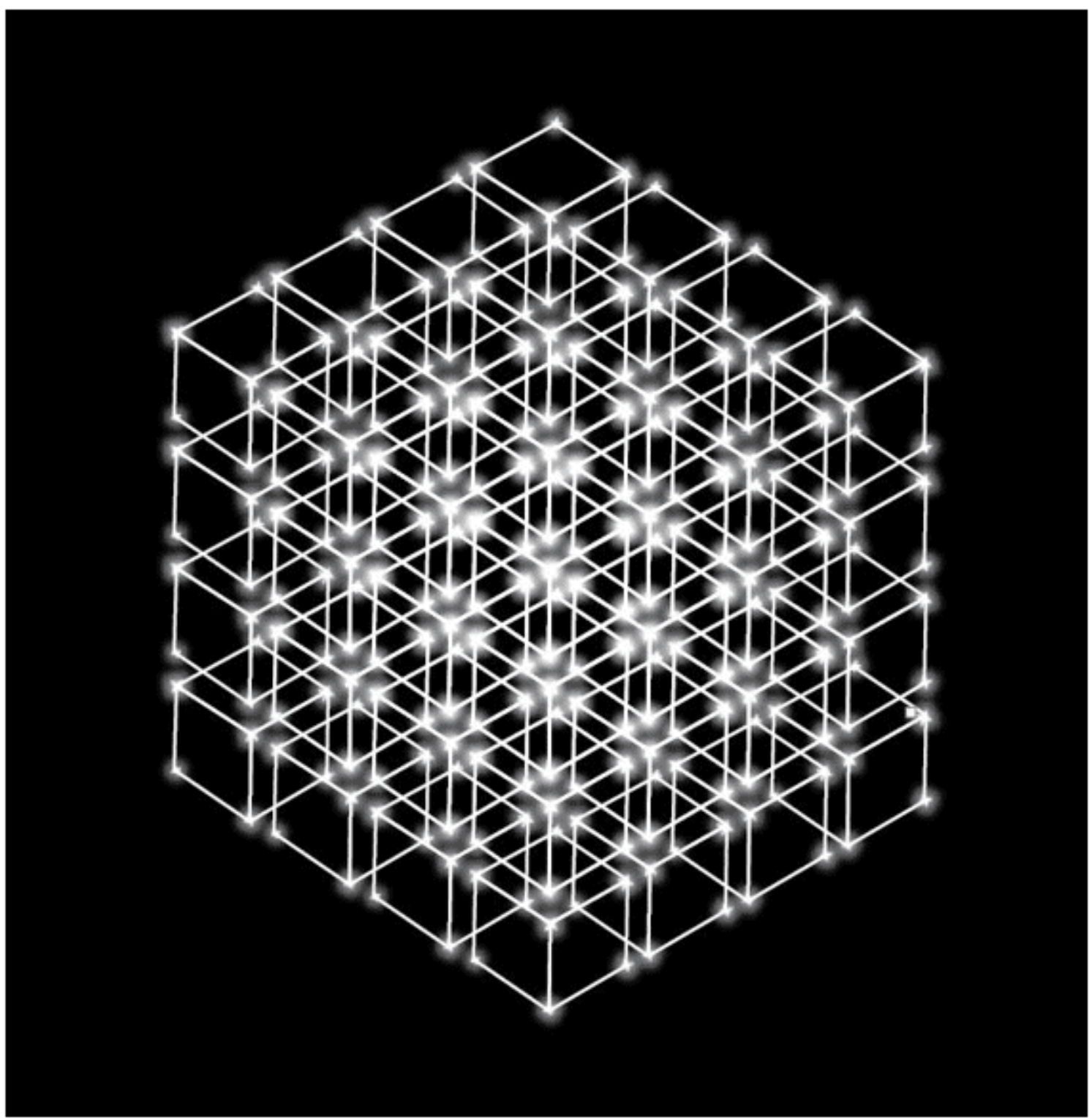

Rappresentazione ottica di Dio, particelle di matrice e stami quantistici. Con il PM a riposo agli angoli dell'EQ, che mostra la distanza, la permeabilità e la neutralità prima

RC: 98377

Disponibile in: https://www.nucleodoconhecimento.com.br/fisica-it/chi-e-dio 
dell'eccitazione gravitazionale, quando il PM con la propria energia e vibrazione radiante sommate formerà massa ed energia, e gli stami formeranno massa, materia oscura e oscurità energia.

\section{APPENDICE - NOTA A PIÈ DI PAGINA DI RIFERIMENTO}

2. Étienne Klein, fisico/filosofo Ph.D. Università École Centrale, nato il 01/04/1958. Nel suo libro II tempo che passa, il fisico sostiene che il futuro esiste già, ma possiamo plasmarlo.

3. Peter Ware Higgs, nato il 29/05/1929, fisico teorico, Università di Edimburgo, Premio Nobel per la fisica, dalla - Particella di Dio - anche se non ha dato questo nome, è stato il suo editore, noto anche come "particella essenziale", il bosone HIGGS è considerato teoricamente come la materia dell'universo.

4. Ludwig Van Beethoven, genio, compositore tedesco del periodo di trascrizione tra classico e romanticismo nascita: 12/1770 Bonn, Germania morte:26/03/1827 Vienna, Austria.

5. Max Karl Ludwig Planck, fisico tedesco, Premio Nobel per la Fisica 1918. Teoria quantistica, fisica quantistica, legge di Planck.

6. Hendrik Brugt Gerhard, fisico olandese, (dal 15/07/1909 al 04/05/2000) evidenziato dalla ricerca "Casimir Effect", Università LEIDEN - Due fluidi superconduttori.

7. Theodor F.E. Kaluza matematico tedesco/polacco (1885/1945), nel 1919 teorizza la gravità quantistica in loop, che oltre alle 3 dimensioni ce n'è una quarta, in un cerchio molto piccolo, e nel 1926, il matematico Oscar Klein (1894/1977) propose la 5 a dimensione che poteva essere estesa o arrotolata, piegata su se stessa, un foglio piegato che salta da un punto all'altro.

Inviato: Febbraio 2021.

$\mathrm{RC}: 98377$

Disponibile in: https://www.nucleodoconhecimento.com.br/fisica-it/chi-e-dio 
Approvato: Settembre 2021.

RC: 98377

Disponibile in: https://www.nucleodoconhecimento.com.br/fisica-it/chi-e-dio 